\title{
The Role of Heart Failure Team in Managing Mechanical Circulatory Support in a Swiss Low-Volume Institution
}

\author{
Piergiorgio Tozzi, MD,${ }^{1}$ Anna Nowacka, MD,${ }^{1}$ Roger Hullin, MD,${ }^{2}$ Patrick Yerly, MD, ${ }^{2}$ \\ Matthias Kirsch, MD $^{1}$ \\ ${ }^{1}$ Cardiac Surgery, Centre Hospitalier Universitaire Vaudois, Lausanne, Switzerland; ${ }^{2}$ Cardiology, Centre Hospitalier \\ Universitaire Vaudois, Lausanne, Switzerland
}

\section{ABSTRACT}

Background: Outcomes after VAD implantation may be dependent on institutional procedural volume. Specifically, it is claimed that high volumes are associated with better clinical results. This study aims to determine whether this procedure is safe even in a low-volume center.

Methods: This is a single-center, retrospective cohort study, including heart failure consecutive patients who received long-term VAD from 2007 to 2017. Primary outcome was survival to transplant or ongoing mechanical circulatory support (MCS) at 1 year. Survival analysis was performed by using the Kaplan-Meier method.

Results: Data concerning 50 adult patients were examined; there were 35 males $(70 \%)$, mean age $49 \pm$ 8 years. VAD was implanted as bridge to transplant (BTT) in 48 and destination therapy (DT) in 2. Devices implanted were HeartMate II (HMII) in 18 (36\%), HeartWare (HW) in 20 (40\%), HeartMate III (HMIII) in $12(24 \%)$. Outcomes were death in 16 (32\%), heart transplantation in $24(48 \%)$, uneventful ongoing support in $10(20 \%)$. Data were analyzed according to pre- and post-heart team creation, and 2 groups of 25 patients were identified: 2007-2013 (mean Interagency Registry for Mechanical Assisted Circulatory Support [INTERMACS] level 3.1) and 2014-2017 (mean INTERMACS level 3.9) showing 1 -year survival of $56 \%$ and $80 \%$, respectively. According to the type of device implanted, 3 groups were identified: HMII = 18 (mean INTERMACS level 2.7), $\mathrm{HW}=20$ (mean INTERMACS level 3.3) and HMIII = 12 (mean INTERMACS level 3.7), showing survival of $52 \%, 78 \%$, and $91 \%$, respectively.

Conclusions: Long-term MCS can be implanted at lowvolume centers with survival rate not inferior to high-volume centers. A heart team specifically trained in heart failure is probably more important than institutional volume in determining outcomes after VAD implantation.

Received February 5, 2018; received in revised form May 24, 2018; accepted May 24, 2018.

Correspondence: Prof Piergiorgio Tozzi, MD, Cardiovascular Surgery Dpt, Rue du Bugnon 46, 1011 Lausanne, Switzerland; (Piergiorgio.Tozzi@chuv.ch).

\section{INTRODUCTION}

In the last 2 decades, several studies have described higher rates of operative mortality with selected surgical procedures at low-volume hospitals, suggesting an inverse correlation between the number of high-risk surgical procedures and mortality [Begg 1998; Birkmeyer 2002; Finks 2011]. The main reason claimed for explaining this inverse correlation is the lack of experience of the surgical team and, more in general, of the low-volume of health care providers in handling complex surgical procedures associated with complex and potentially fatal complications. Although the hospital volume

Table 1. Preoperative Clinical Information of the Patients Who Underwent VAD Implantation

\begin{tabular}{lc}
\hline & VAD patients $(\mathrm{n}=50)$ \\
\hline Male & $35(70 \%)$ \\
Mean age & $49 \pm 8$ \\
Heart failure aetiology & \\
Ischemic cardiomyopathy & $26(52 \%)$ \\
Nonischemic cardiomyopathy & $4(8 \%)$ \\
Acute myocardial infarction & $10(20 \%)$ \\
Idiopathic & $10(20 \%)$ \\
Intention of therapy & \\
BTT & $48(96 \%)$ \\
DT & $2(4 \%)$ \\
Hospital length of stay (days) & $32(21$ to 224$)$ \\
NYHA class IV & $31(66 \%)$ \\
INTERMACS level & 3.2 \\
Cardiac arrest & $8(16 \%)$ \\
Other organ failure & \\
Renal & $13(26 \%)$ \\
COPD & $2(4 \%)$ \\
\hline &
\end{tabular}

BTT, bridge to transplant; DT, destination therapy; INTERMACS, Interagency Registry for Mechanical Assisted Circulatory Support; COPD, chronic obstructive pulmonary disease. 


\section{Heart Failure team structure and roles}

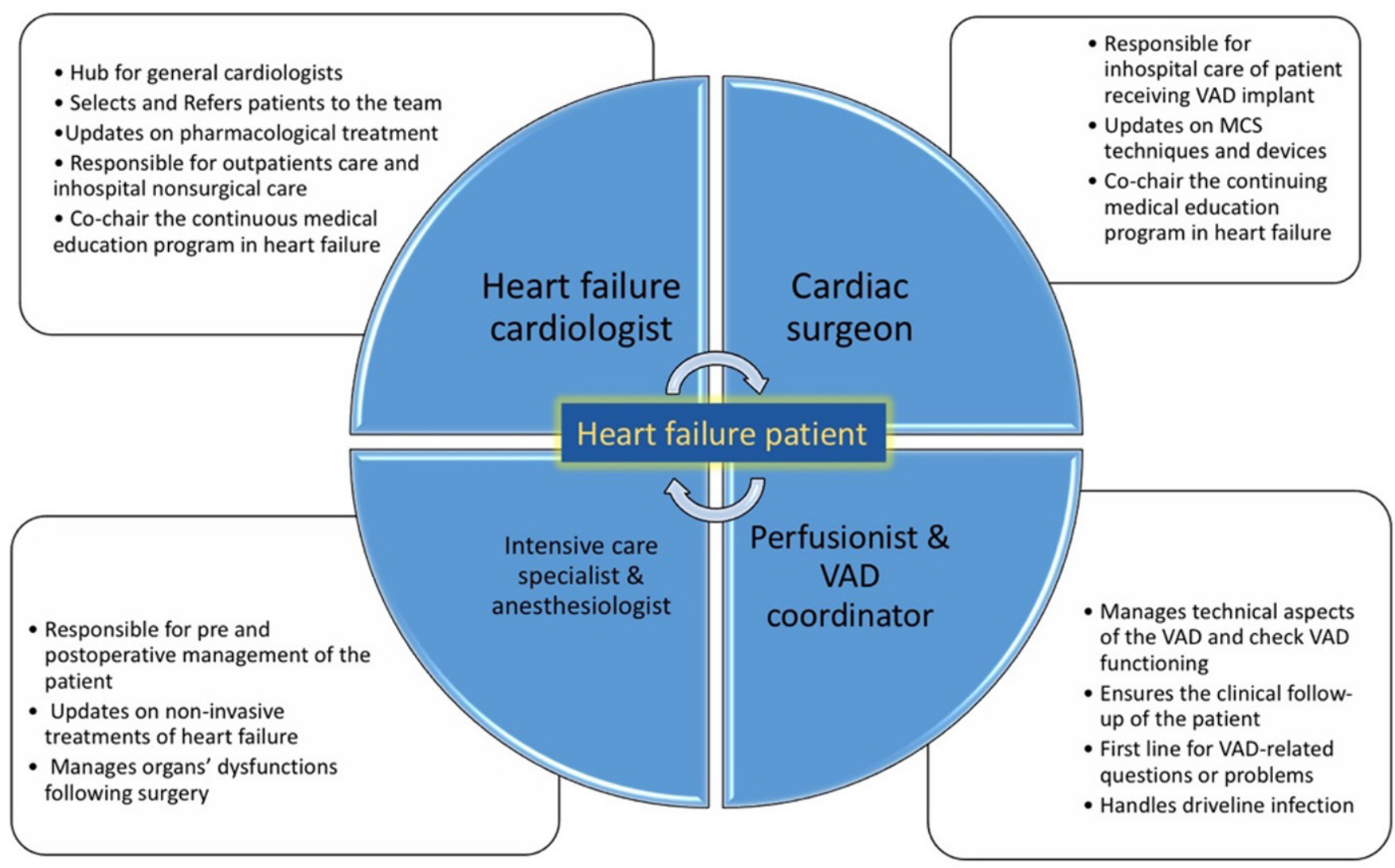

Figure 1. Organization and key roles of the members of the heart failure team.

of a few high-risk cancer procedures (eg, pancreatectomy and esophagectomy) is a strong predictor of operative risk, the relationship between volume and outcome are considerably weaker for cardiac surgical procedures, such as CABGs [Qian 2011]. More specifically, LaPar et al clearly demonstrated that hospital procedure volume is not associated with in-hospital mortality for the performance of CABGs, and they did not find a threshold value for hospital procedure volume at which mortality risk was significantly increased. Patient mortality risk was primarily attributable to patient-level risk factors [LaPar 2012].

Is it the same for patients requiring long-term mechanical circulatory support (MCS)? The first study to investigate the use of long-term MCS was the landmark REMATCH trial, which demonstrated superior survival and quality of life in patients supported with LVAD when compared with those treated medically (52\% versus $23 \%$ 1-year survival) [Rose 2001]. Since then, the number of hospitals accredited to perform MCS proliferated rapidly even in non-heart transplantation centers. In the first 3 years after LVAD therapy approval, in the United States the majority (53\%) of 377 destination therapy (DT) recipients underwent device placement at centers that performed fewer than 4 DT implants
[Lietz 2009]. Lietz et al investigated the effect of center volume on outcomes after VAD implantation and categorized centers as small if they had implanted fewer than 50 devices as bridge to transplant (BTT) or fewer than 4 as DT per year [Lietz 2009].

Rose et al showed that center experience with DT seemed to significantly correlate with the 1-year survival, but the DT center volume was not an independent predictor of 1-year survival with DT when adjusted for the preoperative DT risk score, suggesting that other factors, such as improved candidate selection, may play a role in improving long-term results [Rose 2001]. Further, a systematic review examining the influence of surgery volume on patient outcome determined that individual surgeon volume had a greater effect on outcomes than institutional procedural volume [Chowdhury 2007]. Therefore, the statement that institutional volume accurately represents medical expertise does not always correspond to reality.

The objective of this study is to review the outcomes of patients who were enrolled in our long-term MCS program, to assess whether the LVAD can be safely implanted in a lowvolume, heart transplantation center. 
Table 2. Major Adverse Cardiac Events in the 50-Patient Cohort 30 Days after VAD Implantation*

\begin{tabular}{|c|c|c|c|}
\hline Right ventricular failure requiring mechanical support & $17(34 \%)$ & $11(44 \%)$ & $6(24 \%)$ \\
\hline Bleeding requiring surgery & $19(38 \%)$ & $11(4 \%)$ & $8(32 \%)$ \\
\hline \multicolumn{4}{|l|}{ Stroke } \\
\hline Hemorrhagic & $1(2 \%)$ & $1(4 \%)$ & 0 \\
\hline \multicolumn{4}{|l|}{ Driveline (DL) infection } \\
\hline Conservative treatment & $22(44 \%)$ & $12(48 \%)$ & $10(40 \%)$ \\
\hline DL transposition & $1(2 \%)$ & 0 & 1 \\
\hline Pump thrombosis & $1(2 \%)$ & $1(4 \%)$ & 0 \\
\hline
\end{tabular}

*Raw data are presented.

\section{METHODS}

A low-volume center is a center implanting fewer than 50 devices as BTT or fewer than 4 as DT per year [Lietz 2009].

\section{Study Design}

This is a single-center, retrospective cohort study, examining clinical outcomes of consecutive patients in end-stage heart failure who received a long-term VAD from November 2007 to March 2017, either as BTT or as DT. All patients underwent heart transplant eligibility workup and were enrolled in the heart transplantation and DT program running in our institution. CHUV is a university teaching hospital in which approximately 600 cardiac procedures with extracorporeal circulation are performed annually. From 2007 to 2011, VAD therapy was handled mainly by the general cardiac surgeon and the cardiologist. From 2013 to 2017, patients were systematically discussed in structured heart team meetings including also anesthesiologists, intensive care therapists, and perfusionists, all specifically trained in MCS. The role of VADs coordinators was also created. During the weekly meeting, patients in end-stage heart failure were presented by the cardiologist or by the intensive care specialist, and the different therapeutic options were discussed and analyzed according to the most updated literature. For each patient the team defined a clear strategy of treatment including the level of therapeutic commitment in dealing with complex problems and the role of each specialist in the different phases of the therapeutic project (preoperative, operative, postoperative, and long-term). Roles and competences of each member of the team are illustrated in Figure 1.

\section{Data Collection}

Baseline clinical characteristics, preimplantation clinical course and outcomes were obtained from the medical records. The primary outcome was survival till the transplantation or ongoing MCS at 1 year. Secondary end points were the frequency of major adverse cardiovascular events (MACE) as defined by Kip [Kip 2008].

\section{Statistical Analysis}

Normally distributed continuous variables were reported as mean $\pm \mathrm{SD}$ and compared by using the Student $\mathrm{t}$ test. Survival analysis was performed using the Kaplan-Meier method with censoring for cardiac transplantation. A $P$ value $<.05$ was considered statistically significant.

\section{RESULTS}

During the 10-years study period, 50 adult patients received MCS (Table 1); there were 35 males (70\%), mean age $49 \pm 8$ years. All patients exhibited NYHA IV heart failure symptoms. Causes of heart failure included ischemic cardiomyopathy $(\mathrm{n}=26)$, acute myocardial infarction $(\mathrm{n}=10)$, idiopathic (unknown origin) $(n=10)$, and others $(n=4)$. VAD was implanted as BTT in 48 and DT in 2.

The devices implanted were HeartMate II in 18 (36\%), HeartWare in 20 (40\%), HeartMate III in 12 (24\%). Five patients $(10 \%)$ required temporary right ventricular support with CentriMag pump (St. Jude Medical, St. Paul, MN, USA) because of failure to wean from CPB. After device implantation, antiplatelet therapy was initiated with acetylsalicylic acid, and after drains' removal, anticoagulation was achieved with intravenous heparin followed by transition to anti-vitamin K. From 2013 on, the Heart Failure Team handled the MCS program. Outcomes were death in $16(32 \%)$ (of which 10 were inhospital deaths), heart transplantation in $24(48 \%)$, uneventful ongoing support in $10(20 \%)$ (Figure 2).

The mean waiting time under MCS before transplantation was $316 \pm 61$ days. Data were analyzed according to the management team (pre- and post-heart team era), and 2 groups of 25 patients were identified: 2007-2013 (mean Interagency Registry for Mechanical Assisted Circulatory Support [INTERMACS] level 3.1) and 2014-2017 (mean INTERMACS level 3.9), showing survival at 1 year of $56 \%$ and $80 \%$, respectively (Figure 3 ). 
Overall survival under long-term MCS in the 50-patient cohort

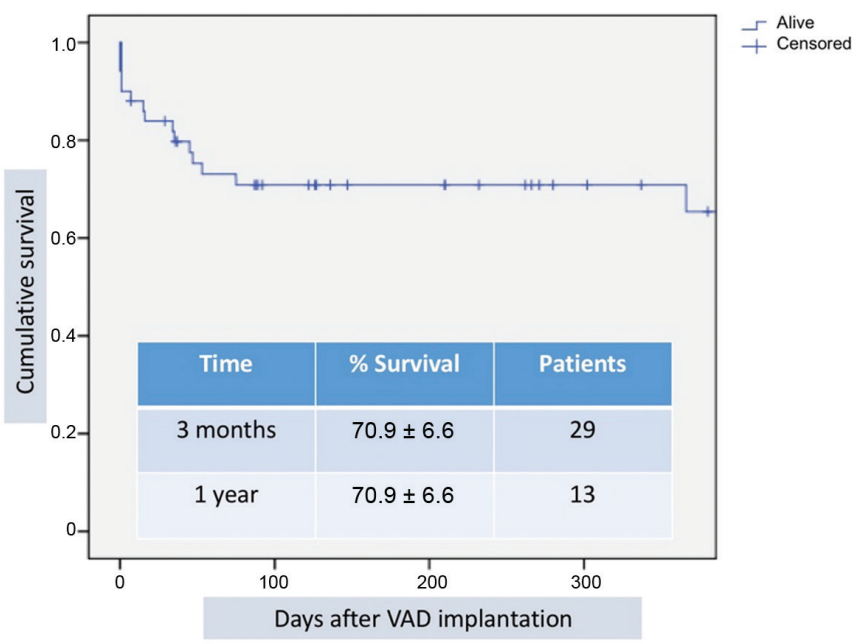

Figure 2. Cumulative survival of patient under VAD according to implantation date.

According to the type of device implanted, 3 groups of patients were identified: HeartMate II (HMII) $=18$ (mean INTERMACS level 2.7), HeartWare $(H W)=20$ (mean INTERMACS level 3.3) and HeartMate III (HMIII) $=12$ (mean INTERMACS level 3.6), showing survival at 1 year of $52 \%, 78 \%$, and $91 \%$, respectively (Figure 4). MACE are illustrated in Table 2.

\section{DISCUSSION}

The need for MCS in patients waiting for heart transplantation is dramatically increasing in Switzerland [Tozzi 2016]. In the last 10 years, the number of patients waiting for heart transplantation has increased by $120 \%$, whereas the number of patients receiving transplantation remains stable (in 2016, 41 received an organ, and 150 were on the waiting list). We conducted this study to assess whether LVAD can be safely implanted in a low-volume, heart transplantation center. At the beginning of our experience, the patient was referred to a surgeon either when the patient was in cardiogenic shock or was deteriorating on inotropes (INTERMACS profiles 1 and 2). VAD implantation was considered as the last "lifesaving" treatment; and, in such condition, the discussion on the patient selection was unrealistic. Moreover, anesthesiologists and intensive care specialists were not specifically trained for managing chronic heart failure patients with VADs. Clinical results were poor and thus encourage cardiologists to defer referral. Late referrals, when patients are too sick to tolerate the LVAD surgery, further perpetuate the vicious cycle of serious operative complications, poor outcomes, and the reluctance to extend such treatment to healthier populations. The survival rate in the pre-heart team era was below $60 \%$ at 1 year. We therefore decided to build a heart team dedicated to heart failure also involving specialists in other domains than cardiac surgery and cardiology with specific

\section{Survival under MCS according to management strategy}

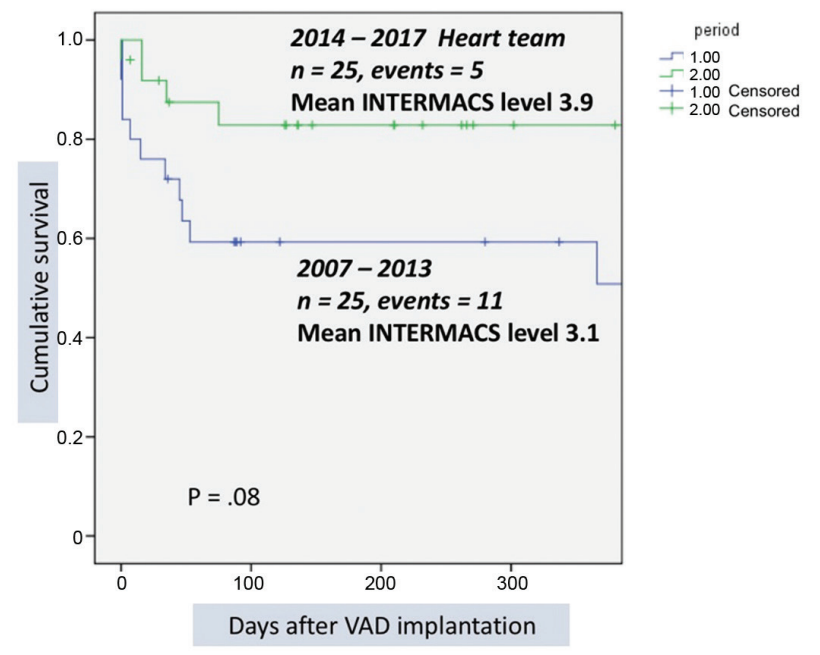

Figure 3. Cumulative survival of the 2 cohorts of patients managed with and without team approach.

training in heart failure patients. This approach was independent of the number of patients treated per year that remained constantly below 20. It is well-known that multidisciplinary and structured team work enhances the quality of care, and we believe this is even more important for the management of patients requiring VAD therapy given the complexity of the technology employed, the critically ill population, and the intensive long-term postoperative medical therapy required. Chowdhury and colleagues have shown that surgeon specialty training and the contribution of specialty-trained members of a multidisciplinary team responsible for patient care are independently associated with improved patient outcomes [Chowdhury 2007]. Our team also included anesthesiologists, intensive care specialists, specialized nurses, perfusionists, and VAD coordinators (Figure 1).

Each team member received specific training in VAD therapy in high-volume centers, in attending dedicated workshops and courses endorsed by the EACTS, in wet labs, and in meetings. They also participate in continuing medical educational program in MCS provided by national and international medical associations or supported by industry. The first positive effect of the heart team approach was on patient selection. The traditional resistance to referring patients with end-stage heart failure earlier in the disease course was mitigated by directly involving the heart-failurespecialist cardiologists in the MCS program. Since then, the number of "crash and burn" patients reduced dramatically (from 10 to 5), and the mean INTERMACS level of the patients treated from 2014 to 2017 was higher than that of the previous group (Figure 3). The surgical procedure did not change significantly except for the number of temporary mechanical supports implanted to assist the right heart. The other aspect concerns medical management in the immediate postoperative phase. Intensive care specialists and cardiologists shared the experience they acquired in high-volume 


\section{Survival under MCS according to device}

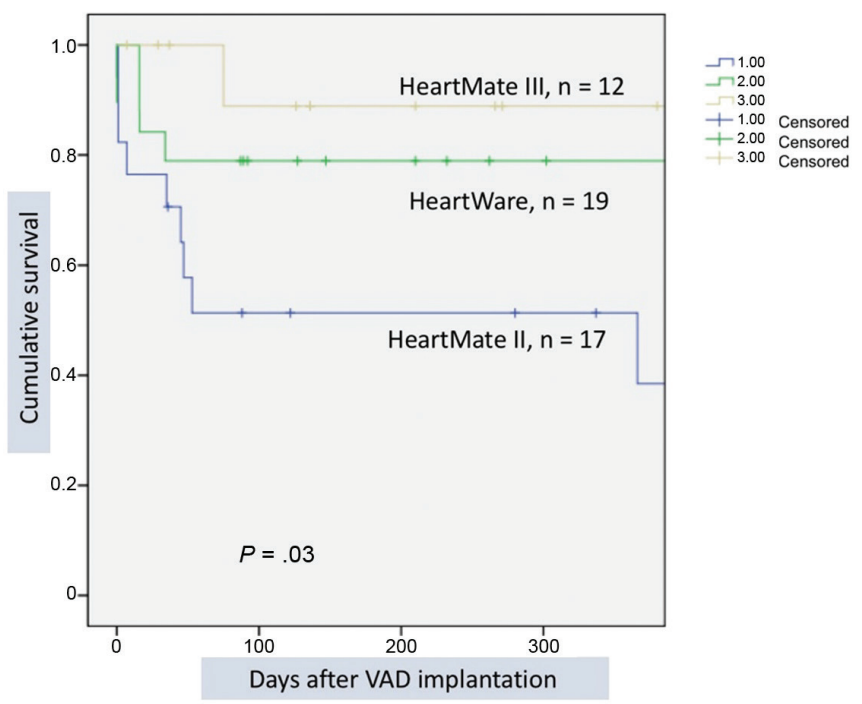

Figure 4. Survival rate according to device implanted. There is no statistical difference in survival rate between HMIII and HeartWare.

centers on hemodynamic optimization, including fluid and inotrope therapy, VAD settings, and support of right ventricular function. Echocardiography has become an essential tool in optimizing hemodynamics, identifying complications, and predicting right ventricular failure [Atluri 2013], and all treatment adjustments are done under echocardiographic control.

The introduction of the VAD coordinator also played a key role in improving long-term results that largely pertain to prevention and treatment of infectious complications, the main cause of death with DT [Lietz 2007]. Two studies comparing early- to late-enrolment in the REMATCH trial [Park 2005; Lietz 2007] and outcomes at the 4 largest-volume US centers pointed to infection as the single complication, the rates of which significantly decreased as center experience increased. The 1-year and 2-year prevalence rates of driveline (DL) infection were, respectively, 9\% and 19\% [Dean 2015]. Our (DL) infection rate was significantly higher than that reported in the literature, but the clinical impact was limited to daily wound care for all patients except one who required cable transposition.

The one-year survival rate of patients treated by use of the multidisciplinary approach was noninferior to the best clinical results reported in the literature [Takeda 2014].

The MOMENTUM 3 trial has recently shown that the fully magnetically levitated centrifugal pump HeartMate 3 has a higher rate of survival free of stroke or reoperation to replace the pump at 6 months after implantation than was implantation of the mechanical-bearing axial continuous flow pump HeartMate II among patients with advanced heart failure, irrespective of their eligibility for transplantation [Mehra 2017]. These results are consistent with the results of another centrifugal LVAD, the HeartWare HVAD. In a recent report, Schmitto and al show excellent outcomes for patients on the device, with a survival rate of almost $60 \%$ at 5 years [Schmitto
2016]. We, therefore, believe that the improvement in our long-term results is also due to the technical performances of new-generation magnetically levitated pumps (Figure 4).

Our study shows all the limitations of a low-volume center. The methodology lacks solid scientific approach, given the retrospective study design, and the small sample size limits the possibility to compare outcomes among patient subgroups. It is not possible to analyze statistical differences among subgroups and clearly identify the determinants of the outcomes.

There have been a number of devices used each in relatively small numbers, and this is in keeping with improvements in technology and advancing knowledge. It makes longitudinal comparison based on device extremely hard.

In conclusion, in this article we share our experience and stress the importance of teamwork even if this is not supported by statistical analysis. We believe that the institutional expertise in VAD therapy has a significant impact on outcomes of this therapy, but at least in our hands, is not correlate to caseload. Long-term MCS can be implanted at low-volume centers, with survival rate not inferior to most recent clinical trials. Although we were not able to elucidate which aspects of the experience were the most critical, better selection of candidates, systemic approach to surgical and postoperative care, as well as the long-term medical management, may have all contributed to the improved outcomes.

Availability of a trained heart team with expertise in longterm MCS treatment facilitates appropriate patient selection and hopefully prevents the futile use of VAD therapy, which can have a devastating impact on families and supporting clinicians when expectations of survival are unrealistic. A heart team specifically trained in heart failure is probably more important than institutional volume in determining outcomes after VAD implantation.

\section{REFERENCES}

Atluri P, Goldstone AB, Fairman AS, et al. 2013. Predicting right ventricular failure in the modern, continuous flow left ventricular assist device era. Ann Thorac Surg 96(3):857-63.

Begg CB, Cramer LD, Hoskins WJ, Brennan MF. 1998. Impact of hospital volume on operative mortality for major cancer surgery. JAMA 280:1747-1751.

BirkmeyerJD, Siewers AE, Finlayson EV, et al. 2002. Hospital volume and surgical mortality in the United States. N Engl J Med 346(15):1128-37.

Chowdhury MM, Dagash H, Pierro A. 2007. A systematic review of the impact of volume of surgery and specialization on patient outcome. Br J Surg 94:145-61.

Dean D, Kallel F, Ewald GA, et al. 2015. Reduction in driveline infection rates: results from the HeartMate II Multicenter Driveline Silicone Skin Interface (SSI) Registry. J Heart Lung Transplant 34(6):781-9.

Finks JF, Osborne NH, Birkmayer JD. 2011. Trends in hospital volume and operative mortality for high risk surgery. N Engl J Med 364:2128-37.

Kip K. 2008. The problem with composite end points in cardiovascular studies: the story of major adverse cardiac events and percutaneous coronary intervention. J Am Coll Cardiol 51(7):701-7.

LaPar DJ, Kron IL, Jones DR, Stukenborg GJ, Kozower BD. 2012. Hospital procedure volume should not be used as a measure of surgical 
quality. Ann Surg 256(4):606-15.

Lietz K, Long JW, Kfoury AG, et al. 2007. Outcomes of left ventricular assist device implantation as destination therapy in the post-REMATCH era: implications for patient selection. Circulation 116:497-505.

Lietz K, Long JW, Kfoury AG, et al. 2009. Impact of center volume on outcomes of left ventricular assist device implantation as destination therapy: analysis of the Thoratec HeartMate Registry, 1998 to 2005. Circ Heart Fail 2(1):3-10.

Mehra MR, Naka Y, Uriel N, et al. 2017. A fully magnetically levitated circulatory pump for advanced heart failure. N Engl J Med 376(5):440-450.

Park SJ, Tector A, Piccioni W, et al. 2005. Left ventricular assist devices as destination therapy: a new look at survival. J Thorac Cardiovasc Surg 129(1): 9-17.

Qian F, Lustik SJ, Diachun CA, Wissler RN, Zollo RA, Glance LG.
2011. Association between Leapfrog safe practices score and hospital mortality in major surgery. Med Care 49(12):1082-8.

Rose EA, Gelijns AC, Moskowitz AJ, et al. 2001. Long-term use of a left ventricular assist device for end-stage heart failure. N Engl J Med 345(20):1435-43.

Schmitto JD, Zimpfer D, Fiane AE, et al. 2016. Long-term support of patients receiving a left ventricular assist device for advanced heart failure: a follow-up analysis of the Registry to Evaluate the HeartWare Left Ventricular Assist System. Eur J Cardiothorac Surg 50(5):834-838.

Takeda K, Takayama H, Kalesan B, et al. 2014. Long-term outcome of patients on continuous-flow left ventricular assist device support. J Thorac Cardiovasc Surg 148(4):1606-14.

Tozzi P, Hullin R. 2016. Mechanical circulatory support for destination therapy. Swiss Med Wkly 146:w14267. 